\title{
Eops: Duloxetina para el tratamiento de la fibromialgia en pacientes con depresión mayor
}

\author{
Duloxetine for the treatment of fibromyalgia in patients with major depression
}

Juan I. Trobbiani

\begin{abstract}
Resumen
Se presenta el caso de una paciente con diagnóstico de fibromialgia y depresión mayor de larga data. Se evalúa críticamente una revisión sistemática y metaanálisis donde se discuten estrategias farmacológicas en pacientes con esa condición clínica. Se concluye que la literatura basada en evidencia para elegir la droga adecuada para el paciente individual es de baja calidad y no permite emitir recomendaciones bien fundamentadas sobre alguna opción en particular.

\section{Abstract}

The case of a patient with major depression and fibromyalgia is presented. A systematic review and metaanalysis on therapeutic options in patients with that clinical condition is critically appraised. The conclusión is that evidence-based literature on the topic is low-quality and that it does not allow well-founded recommendations to be made on the preferred drug treatment.
\end{abstract}

Palabras clave: Fibromialgia - Depresión - Terapéutica. Key words: fibromyalgia, depression, theraphy.

Trobbiani. Duloxetina para el tratamiento de la fibromialgia en pacientes con depresión mayor. Evid Act Pract Ambul. 2014:17(3).Jul-Sep. 107-109.

\section{Caso clínico}

Concurre al consultorio de atención primaria una paciente de 64 años con antecedentes de depresión mayor de larga data, fibromialgia, hipotiroidismo y colon irritable. Polimedicada con trimebutina (200mg/día), omeprazol (40mg/día), clonazepam (4 $\mathrm{mg} /$ día), sertralina (50mg/día), levotiroxina (75mg/día), gotas oftálmicas y analgésicos a demanda (ibuprofeno / diclofenac).

No tiene antecedentes familiares de relevancia. Tiene dos hijos, vive sola y tiene un seguro de salud. Trabaja como ama de casa y practica yoga. Consulta por padecer dolor crónico en el hombro, al que se suman dolores generalizados en otras partes del cuerpo. En su última cita con el médico reumatólogo, éste le prescribió duloxetina.

\section{Pregunta}

En pacientes adultos con fibromialgia y depresión mayor, ¿la duloxetina ofrece ventajas para el control del dolor, comparada con el tratamiento farmacológico estándar de la fibromialgia?

\section{Búsqueda}

Se realizaron dos búsquedas diferentes con estrategias similares. La primera en Medline a través de PubMed con la siguiente estrategia: ("Fibromyalgia"[Mesh]) AND duloxetine, filtros: Systematic Review, Spanish, English. La segunda en Epistemonikos, con la estrategia: "fibromyalgia" AND "depression" AND "duloxetine", filtros: Systematic Review. Se detectó un artículo que abordaba la pregunta en cuestión.

\section{Aspectos relevantes de la fibromialgia}

La fibromialgia es un problema frecuente en atención primaria. Representa el segundo motivo de consulta reumatológica después de la osteoartritis. Es más frecuente en mujeres $(6: 1)$. Sus síntomas principales son dolor crónico generalizado, asociado con disfunción cognitiva, trastornos del sueño y fatiga física, sobre todo matinal. Frecuentemente se asocia a depresión así como a otros trastornos (cefalea tensional, fatiga crónica, bruxismo, colon irritable y vejiga irritable). La fisiopatología es desconocida. Actualmente uno de los focos de investigación está en ciertas alteraciones del procesamiento del dolor en el sistema nervioso central (SNC).
El diagnóstico de fibromialgia se realiza según los criterios del Colegio Americano de Reumatología ${ }^{1}$, en función de una historia de síntomas típicos y la exclusión de causas somáticas de enfermedad. No existen pruebas de laboratorio específicas. Los objetivos del tratamiento de la fibromialgia son el alivio de los síntomas y la mejora en la calidad de vida. Las medidas terapéuticas usuales en pacientes con fibromialgia comprenden la educación, la adherencia a un programa de actividad física [ejercicio aeróbico, estiramiento y fortalecimiento] y el tratamiento farmacológico ${ }^{2}$.

Los fármacos más estudiados y que han demostrado ser efectivos en el tratamiento de la fibromialgia son los antidepresivos y algunos anticonvulsivantes (pregabalina). Los antidepresivos clásicos son los tricíclicos (amitriptilina, desipramina) y los Inhibidores Selectivos de la Recaptación de Serotonina (ISRS) [fluoxetina, sertralina]. Más recientemente se ha estudiado otro grupo de antidepresivos, los Inhibidores Selectivos de la Recaptación de Serotonina y Noradrenalina (ISRSN) [duloxetina, milnazipram] y se estableció su seguridad y eficacia en el tratamiento de la fibromialgia ${ }^{3,4,5}$.

Los ISRSN son antidepresivos que aumentan las concentraciones de serotonina y noradrenalina en el cerebro. La acción antiálgica de los ISRSN parece deberse a ese incremento de la actividad endógena de los sistemas descendentes inhibidores del dolor, que son de carácter noradrenérgico y serotoninérgico. Estos sistemas podrían cumplir un papel importante en la fisiopatología de la fibromialgia. Los ISRSN han sido aprobados por la US FDA (Federal Drug Administration) para el tratamiento de la fibromialgia, pero han sido rechazados por su par europeo (European Medical Agency) por considerar que sus efectos eran demasiado pequeños para ser relevantes para los pacientes.

En el tratamiento de los pacientes con fibromialgia, típicamente se inicia la terapéutica con alguno de estos fármacos (en general un tricíclico) a baja dosis, que se titula gradualmente hasta alcanzar dosis terapéuticas. En pacientes con fatiga severa/depresión existe consenso generalizado en comenzar con un ISRSN (duloxetina), pero es incierto el grado de evidencia para apoyar esa recomendación.

\section{Resumen de la evidencia}

Se detectó un metaanálisis.

Häuser $\mathbf{W}$ y col. ${ }^{\ddagger}$ The role of antidepressants in the management of fibromyalgia syndrome: a systematic review and meta- 
analysis. CNS Drugs. 2012 Apr 1;26(4):297-307.

\section{$\underline{\text { Validez interna }}$}

\section{Diseño y objetivos}

La revisión fue realizada siguiendo las guías PRISMA. El metaanálisis se desarrolló siguiendo las recomendaciones de la colaboración Cochrane. Su objetivo fue proveer un análisis cuantitativo de la eficacia y los efectos adversos de los antidepresivos en el manejo de la fibromialgia en adultos. La pregunta del estudio es clara, aunque incluye varias drogas y diferentes medidas de resultados. Una de ellas responde la pregunta generada.

\section{Estrategia de búsqueda}

Los autores ampliaron las estrategias de búsqueda utilizadas en dos revisiones previas ${ }^{6,7}$ en las bases MEDLINE, SCOPUS y la base Cochrane de Registro de Ensayos Clínicos. Revisaron la lista de referencias de los artículos incluidos y también literatura gris. Sin embargo, no informaron sobre restricciones de idiomas ni detallaron la estrategia de búsqueda. En síntesis, el acceso a la información es posible, pero resulta incómodo porque hay que buscarla en trabajos previos de los mismos autores ${ }^{6,7}$.

\section{Criterios de inclusión y exclusión}

Ensayos Clínicos Randomizados (ECA), que hubieran comparado cualquier tipo de antidepresivo con placebo o con otros antidepresivos. Para ser seleccionados en la revisión los estudios debían haber incluido pacientes con diagnóstico de fibromialgia y haber evaluado en ellos alguno de los siguientes dominios: dolor, sueño, fatiga, calidad de vida relacionada con la salud (health-related quality of life, $\mathrm{HRQOL}$ ). Adicionalmente, debían haber reportado tasas de abandono de tratamiento debidas a efectos adversos. Fueron excluidos los estudios en los que la intervención había sido una combinación de antidepresivos con otro tratamiento y aquellos en los que la comparación hubiera sido no farmacológica.

\section{Estrategias de minimización de sesgos}

Se analizó la calidad de los artículos incluidos con la escala van Tulder $^{8}$. Dos autores independientemente extractaron los datos en formularios estandarizados. Las discrepancias se resolvieron por consenso. Cuando fue necesario, un tercer autor revisó los datos para llegar a un consenso. Se contactó a autores de los ensayos para completar la información faltante. $\mathrm{Al}$ igual que el resto de la información sobre "metodología", el acceso es posible, pero es necesario referirse a revisiones previas ${ }^{6,7}$. Los autores no presentan gráficos de dispersión de los estudios incluidos.

\section{Análisis de datos}

Se utilizó la diferencia de medias estandarizada para comparar los resultados del grupo placebo con los del grupo tratamiento. Se utilizaron las categorías de Cohen para evaluar la magnitud del tamaño del efecto. Para el meta-análisis, se evaluó la heterogeneidad con la prueba de $\mathrm{I} 2(>50 \%$ indicaba heterogeneidad fuerte) y se utilizó un modelo de efectos aleatorios.

\section{Resultados}

Fueron incluidos 35 estudios en el metaanálisis, 12 referidos a ISRSN (6070 pacientes). La duración promedio de los estudios de ISRSN fue de 15,5 semanas (rango: 12 a 28). La calidad de los estudios fue moderada (media de escala van Tulder 7.5 [rango: 5 a 8] para un máximo de 11). No puede accederse al flujograma de los estudios incluidos y tampoco se describen en detalle los estudios incluidos en la revisión.

Los estudios con duloxetina incluyeron pacientes con depresión mayor y todos los estudios excluyeron pacientes con otros trastornos depresivos o ansiosos. Todos los ensayos realizaron análisis por intención de tratar. Las diferencias de medias para dolor, sueño, fatiga, depresión y calidad de vida con el uso de Inhibidores Selectivos de la Recaptación de Serotonina y Noradrenalina (ISRSN) fueron estadísticamente significativas. Sin embargo, basados en las categorías de Cohen, el tamaño del efecto fue pequeño para el dolor y no fue sustancial para el sueño, la fatiga, la depresión y la calidad de vida HRQOL. El NNT para una reducción del $30 \%$ del dolor fue de 10, (IC 95; 8 a 13). La heterogeneidad de los estudios fue baja $\left(I^{2}=4 \%\right)$.

Validez externa ¿Los resultados de los autores se aplican a mi paciente?

La paciente que motivó la búsqueda posee una cobertura de salud de gama baja-media que no reconocería la prestación y es poco probable que pueda afrontar ese gasto de su bolsillo pues sus ingresos económicos son limitados y según el sitio Kairos, la duloxetina cuesta aproximadamente 10 veces más que la amitriptilina.

\section{Comentario final}

La eficacia de los antidepresivos en pacientes con fibromialgia ha sido probada en ensayos controlados y meta-análisis ${ }^{3-6}$, pero no existen comparaciones directas entre ellos ni con drogas previas. Los resultados que presentan los autores no son de alto impacto y aportan poco a lo que se desprende de los estudios primarios. Sin embargo y a pesar de ello, los autores concluyen que la amitriptilina y los ISRSN duloxetina y milnazipram son opciones de primera línea para el tratamiento de la fibromialgia. Adicionalmente, establecen que la amitriptilina debería ser el fármaco de elección cuando los pacientes asocian trastornos del sueño y la duloxetina, cuando existe depresión mayor como comorbilidad. Señalan no obstante, que los pacientes y médicos deberían ser realistas y moderar las expectativas respecto a los beneficios potenciales de los antidepresivos en la fibromialgia. Un pequeño número de pacientes experimentan importante alivio de síntomas con pocos o ningún efecto adverso, aunque no es despreciable el número de pacientes que experimentan un pequeño beneficio o que deben abandonar la terapia por sus efectos adversos.

Vale destacar que la calidad del artículo seleccionado es moderada-baja, pero no desentona con el resto de la literatura en el tema, escasa y sin estudios bien diseñados. Los factores que contribuyen a esta situación son de distinto orden: 1) La fisiopatología de la fibromialgia y la depresión, así como los mecanismos de acción de las drogas no son del todo conocidos. 2) Los individuos incluidos en las investigaciones publicadas no han tenido seguimiento prolongado (ninguno superó los seis meses). 3) El diagnóstico diferencial es dificultoso (depresión vs. fibromialgia vs síndromes de dolor miofascial) debido a la

¥ Winifried Häuser es médico clínico y psicosomático alemán, una autoridad mundial en la materia, tiene indexados más de 200 trabajos en Medline como primer autor, 45 de ellos con el descriptor MeSH "Fibromialgia". Es autor de las últimas revisiones sistemáticas y metaanálisis importantes sobre el tema. Tiene algunos vínculos profesionales con empresas farmacéuticas. 
clínica amplia de la depresión, a los signos y síntomas inespecíficos de los síndromes somáticos funcionales y a la notable comorbilidad en estos pacientes. Consideramos importante ser lo más certeros posibles y hacer un esfuerzo en diferenciar si se trata de una paciente con "depresión por dolor crónico" vs una paciente con la comorbilidad "fibromialgia más depresión". Asimismo, sería importante volver a indagar la historia clínica de su depresión (¿Cuántos años de evolución? ¿Con qué tratamiento? ¿A qué fármacos respondió? ¿A qué dosis?). 4) Existen fuertes posibilidades de que los autores hayan sido presionados por la industria farmacéutica, dado sus vínculos profesionales y comerciales.

En definitiva, y considerando el estado actual del conocimiento, en pacientes con fibromialgia (tengan o no depresión mayor como comorbilidad) para lograr el objetivo terapéutico de reducir el dolor y mejorar la calidad de vida lo ideal es realizar un esquema terapéutico integral en el que se incluyan fundamentalmente estrategias no farmacológicas (de probada efectividad y escasos/nulos efectos adversos) y farmacológicas. Actualmente no existen evidencias contundentes y de alta calidad para preferir la duloxetina por encima de los fármacos habituales como primera línea en el tratamiento de pacientes con fibromialgia y depresión mayor. En todo caso, deberá elegirse la medicación para el paciente individual en función de la experiencia clínica, la historia, las preferencias del paciente, los síntomas acompañantes (fatiga, insomnio, depresión), los efectos adversos y los costos.

Recibido el 30/09/13 y aceptado el 29/10/14.

\section{Referencias:}

1. Wolfe F y col. The American College of Rheumatology 1990 Criteria for the Classification of Fibromyalgia. Report of the Multicenter Criteria Committee. Arthritis Rheum. 1990 Feb;33(2):160-72.

2. Goldenberg D. Initial treatment of fibromyalgia in adults. In: UpToDate, Basow, edited by Peter H Schur, UpToDate $囚$, Waltham, MA, 2013.

3. Arnold $\mathrm{L}$ y col. A randomized, double-blind, placebo-controlled trial of duloxetine in the treatment of women with fibromyalgia with or without major depressive disorder. Pain. 2005 Dec 15;119(1-3):5-15

4. Arnold $\mathrm{L}$ y col. A double-blind, multicenter trial comparing duloxetine with placebo in the treatment of fibromyalgia patients with or without major depressive disorder. Arthritis Rheum. 2004 Sep;50(9):2974-84.

5. Russell I y col. Efficacy and safety of duloxetine for treatment of fibromyalgia in patients with or without major depressive disorder: Results from a 6-month, randomized, double-blind, placebo-controlled, fixed-dose trial. Pain. 2008 Jun;136(3):432-44

6. Häuser $\mathrm{W}$ y col. Comparative efficacy and acceptability of amitriptyline, duloxetine and milnacipran in fibromyalgia syndrome: a systematic review with meta-analysis. Rheumatology (Oxford) 2011; 50: 532-43

7. Häuser W y col. Treatment of fibromyalgia syndrome with antidepressants: a metaanalysis. JAMA 2009; 301: 198-209

8. Van Tulder M y col. Updated method guidelines for systematic reviews in the cochrane collaboration back review group. Spine. 2003 Jun 15;28(12):1290-9. 